\title{
Gestión Administrativa y ejecución presupuestaria de la Coordinación Zonal de Educación - Zona 3
}

Fecha de recepción : 2020-04-02 • Fecha de aceptación: 2020-06-17 • Fecha de publicación: 2020-09-10

Tupac Amaru Masaquiza Jerez ${ }^{1}$ Universidad Técnica de Ambato, Ecuador tupacmjerez@gmail.com

https://orcid.org/0000-0001-6010-1856

Adrián Marcelo Palacios Ocaña ${ }^{2}$ Universidad Técnica de Ambato, Ecuador ampo1991@gmail.com https://orcid.org/0000-0001-6293-7622

Klever Armando Moreno Gavilánes ${ }^{3}$ Universidad Técnica de Ambato, Ecuador kleveramoreno@uta.edu.ec https://orcid.org/0000-0001-9870-8821

\section{Resumen}

El objetivo de la presente investigación se centra en determinar el nivel de cumplimiento de la partida presupuestaria asignada a la Coordinación Zonal de Educación Zona 3 durante el año 2018, para de esta forma garantizar una educación de calidad a la comunidad, en especial a los niños y adolescentes, quienes son los beneficiarios directos de este servicio; para ello, la metodología utilizada se fundamentó bajo un enfoque cuantitativo, dado que fue necesario procesar información proveniente de documentos oficiales. A partir de ello, y como principales hallazgos, se pudo determinar que la institución al 31 de diciembre de 2018 registró únicamente un 96\% de ejecución presupuestaria; no obstante, a pesar de no cumplir el 100\% de ejecución, la gestión desarrollada se enmarca en un nivel de asignación presupuestaria óptima. 
Palabras clave: ciclo presupuestario, ejecución presupuestaria, gestión administrativa, inversión pública, planificación financiera.

\section{Abstract}

The objective of this research is focused on determining the level of compliance with the budget line assigned to the Zonal Coordination of Education Zone 3 during the year 2018, and thus guarantee quality education to the community, especially children and adolescents who are the direct beneficiaries of this service; For this, the methodology used is based on a quantitative approach since it was necessary to process information from official documents. Based on this and as main findings, it was possible to determine that the institution as of December 31, 2018 registered only $96 \%$ of budget execution, however, despite not complying with $100 \%$ of execution, the management carried out is framed at a level optimal budget allocation.

Keywords: budget cycle, budget execution, administrative management, public investment, financial planning. 


\section{Introducción}

La administración se ha convertido en un fenómeno relevante en el mundo moderno, al surgir dentro de la organización la necesidad de coordinar actividades, tomar decisiones, direccionar al personal y evaluar el desempeño, tomando como parámetro el logro de objetivos previamente definidos. Dicho en otras palabras, se convierte en el timón del barco empresarial que dirige de forma equilibrada todas las acciones dentro de la empresa para llegar a su destino (Falconi, Luna, Sarmiento y Andrade, 2019).

Esta gestión tuvo su origen en la antigüedad, surgió como una necesidad en los primeros tiempos desde la existencia del hombre, cuando los seres humanos tuvieron que juntarse para realizar distintas actividades de sobrevivencia, toma de decisiones, administración de recursos, búsqueda de alimentos, entre otros. Sin embargo, desde los años 1800 se ha convertido en un hábito el hecho de definir a la gestión administrativa con base a cuatro pilares fundamentales: 1) planeación, 2) organización, 3) dirección y 4) control. Estos procesos responden a un ciclo de operaciones que se retroalimentan de forma directa, encaminando los esfuerzos de quienes son parte de la organización hacia la consecución de los objetivos establecidos por la organización (Solano, Merino y Uc, 2016).

La interacción y concatenación de cada uno de estos procesos forman la denominada gestión administrativa, la cual es considerada como una de las actividades con mayor relevancia dentro de la organización, dado que se convierte en un soporte administrativo para los diferentes procesos de la empresa (Marco et al., 2016). Dicho esto, la implicación de llevar una gestión o administración en una organización se puede precisar que representa un factor preponderante al instante de crear un negocio, dado que, una buena o mala dirección determinará en gran medida el éxito o fracaso de una organización.

Por otro lado, en la aplicación de las políticas estatales, la gestión administrativa forma parte de los procesos conocidos como habilidades de apoyo de la organización, por lo que en la práctica misma su función principal está encaminada a la dirección tanto de los recursos humanos, tecnológicos, materiales, y de servicios administrativos; coordinando además los planes, proyectos y programas necesarios para el desarrollo de los distintos procesos institucionales direccionados a lograr eficiencia y eficacia en relación al uso de los recursos disponibles de las instituciones públicas (Escobar, Mora y Huilcapi, 2017).

En la actualidad, resulta indispensable saber y comprender la gestión administrativa y financiera de las instituciones del sector público, cumpliendo con los lineamientos en relación a la ejecución del presupuesto y direccionada hacia la consecución de los objetivos institucionales. En este sentido, el ciclo presupuestario es de cumplimiento obligatorio para todos los organismos y entidades del sector público y comprende las siguientes etapas: 1) programación presupuestaria, 2) formulación presupuestaria, 3) aprobación presupuestaria, 4) ejecución presupuestaria, 5) evaluación y seguimiento presupuestario, y 6) clausura y liquidación presupuestaria (Gavilanez, 2016).

Es importante mencionar que toda institución siempre busca su desarrollo dentro de este contexto sistematizado, con la finalidad de tener un adelanto administrativo-financiero y cubrir 
todas las necesidades presupuestarias, por ende, el presupuesto constituye una herramienta de planificación que de una forma determinada integra y coordina las áreas, actividades, departamentos y responsables de una organización, y que expresa en términos monetarios los ingresos, gastos y recursos que se generan en un periodo determinado para cumplir con los objetivos fijados en la estrategia.

En este sentido, el objeto de estudio se encuentra representado por la Coordinación Zonal de Educación Zona 3, la misma que está conformado por las provincias de Tungurahua, Cotopaxi, Chimborazo y Pastaza con 19 direcciones distritales y 183 circuitos educativos. En dicha institución, durante los últimos años, se ha visto una serie de inconvenientes relacionados con la gestión desarrollada por la parte administrativa y financiera que repercute en la planificación del presupuesto. En términos generales, la débil gestión administrativo-financiero de la coordinación genera un cumplimiento parcial de la ejecución presupuestaria, que dicho en otras palabras es un procedimiento, el cual consiste en gastar la totalidad de los valores asignados a la Unidad Ejecutora (coordinación) para mejorar su gestión y cumplir con los objetivos establecidos con seguimiento y supervisión de la Planta Central del Ministerio de Educación; sin embargo, esto no ocurre así, puesto que existen incumplimientos tanto en obras dentro de las instituciones educativas, como en pagos a distintos proveedores.

A partir de lo señalado en el epígrafe anterior, el estudio se justifica por cuanto la parte administrativa incentiva a la entidad y funcionarios de la misma al logro de las diferentes metas propuestas, entre las cuales se encuentra ejecutar el total del presupuesto asignado; dado que todas las instituciones del sector público cada año reciben asignación presupuestaria para ser terminada en el transcurso del mismo, esta se mide constantemente y el porcentaje de ejecución al mes de junio debe llegar al 50\% para alcanzar a cumplir hasta diciembre un 100\% y de esta forma lograr los objetivos planteados (Gavilanez, 2016).

Bajo este contexto, el presente estudio se centra en determinar el nivel de cumplimiento de la ejecución presupuestaria de la Coordinación Zonal de Educación Zona 3 considerando la gestión administrativa, para garantizar una educación de calidad a la comunidad en especial a los niños, niñas, jóvenes y adultos, que son quienes reciben beneficios a través de la inversión pública.

\section{Gestión Administrativa}

La gestión administrativa constituye un marco en el que las personas vinculadas a una organización trabajan hacia la consecución de un objetivo específico donde se evidencia el estilo de trabajo conjunto para cumplir un fin institucional. En este sentido, también puede ser entendida como una constelación organizada de partes conectadas y similares para establecer una entidad común, con la finalidad de lograr el objetivo deseado por parte de la organización.

En tal virtud, tiene como propósito identificar los aspectos requeridos de una actividad para lograr un objetivo específico o un plan. Por lo tanto, deben estar disponibles factores específicos, tales como: un propósito y trabajo en equipo, un grupo de personas que comparten una relación real y participan en el logro de su objetivo (Pacheco, Robles y Ospino, 2018). En consecuencia, debe ser 
realista, dinámico y activo en el logro de objetivos con los mejores métodos y los costos más bajos, creando cooperación entre un grupo de personas que comparten la coordinación de esfuerzos y negocios.

Por consiguiente, esta dirección tiene un carácter sistémico, al ser portadora de acciones coherentemente orientadas al logro de lo establecido, a través del cumplimiento de las funciones clásicas de la gestión en el proceso administrativo. Además, puede ser descrita como la puesta en práctica de cada uno de los procesos de la administración. Realizar un eficiente esfuerzo en capacitación mejora la gestión administrativa y con ello la eficiencia del personal en la institución (Mora et al., 2018). De esta manera, detallaremos aquellos componentes de dicho proceso.

Planificación: constituye la creación de un plan de acción para el futuro, la determinación de las etapas del plan y la tecnología necesaria para implementarlo. Decidir de antemano ¿qué hacer?, ¿cómo hacerlo?, ¿cuándo hacerlo?, y ¿quién debe hacerlo? (Edwards, 2018)developed around 1900 by the French management theorist Henri Fayol (1841lu20131925. Se asigna el camino desde donde se encuentra la organización hasta dónde quiere estar. La función de planificación implica el establecimiento de metas y disponerlos en un orden lógico. Los administradores participan en la planificación a corto y largo plazo.

Organización: una vez que se diseña un plan de acción, los gerentes deben proporcionar todo lo necesario para llevarlo a cabo; incluyendo materia prima, herramientas, capital y recursos humanos. Identificar responsabilidades, agruparlas en departamentos o divisiones, y especificar relaciones organizacionales. La cultura organizativa de innovación de productos o servicios se sujeta con un entorno cognitivo y social, variado de dogmas, valores y conductas compartidas entre los miembros de la organización (Bonilla et al., 2018)nuevos productos, menores costos. La innovación empresarial genera grandes cambios en la organización, sirve de soporte y sinergia para ser esenciales, eficaces y alcanzar sus grandes objetivos en su propio mercado competitivo. La estructura empresarial se encuentra dependiente de los elementos básicos que engloban a la planeación, organización, implementación y el control, para el desarrollo de sus actividades (Bonilla Jurado et al., 2020).

Mando: los gerentes necesitan implementar el plan, deben comprender las fortalezas y debilidades de su personal (García et al., 2018). Dirigir a las personas de una manera que logren los objetivos de la organización requiere una asignación adecuada de recursos y un sistema de apoyo efectivo. La dirección requiere habilidades interpersonales excepcionales y la capacidad de motivar a las personas. Una de las cuestiones cruciales en la dirección es el equilibrio correcto entre las necesidades del personal y la producción (Armijo, 2018).

Coordinación: los gerentes de alto nivel deben trabajar para "armonizar" todas las actividades para facilitar el éxito organizacional, la comunicación es el principal mecanismo de coordinación, sincroniza los elementos de la organización y se debe tener en cuenta la delegación de autoridad, responsabilidad y el alcance del control dentro de las unidades.

Control: el elemento final de la gestión implica la comparación de las actividades del personal con el plan de acción, es el componente de evaluación de la gestión. Es la función de monitoreo 
que evalúa la calidad en todas las áreas y detecta desviaciones potenciales o reales del plan establecido, asegurando un desempeño de alta calidad y resultados satisfactorios mientras mantiene un ambiente ordenado y sin problemas. El control incluye la gestión de la información, la medición del desempeño y la institución de acciones correctivas.

En definitiva, la gestión administrativa puede ser considerada como una forma en la que los esfuerzos humanos se encaminan al logro de un propósito general, además de practicar la autoridad, coordinación y control. Es un proceso que presta especial atención a todas las actividades, tareas deseadas, identifica autoridades, así como también funciones para lograr los objetivos planteados conjuntamente con la resolución de disputas, problemas que enfrenta cada departamento. Además, es un proceso que establece una estructura de roles en la cual se determina las actividades requeridas para lograr un objetivo específico, combinándolos y delegando a una persona encargada de cumplirlos. Asimismo, permite coordinar las relaciones tanto verticales, como horizontales, determinadas en la estructura organizacional (Abdulrahim y Elbashir, 2016).

\subsection{Principios generales de la gestión administrativa}

La gestión es esencial para cualquier organización que desee ser eficiente y lograr sus objetivos, sin alguien en una posición de autoridad, habría una anarquía organizacional sin estructura ni enfoque, asimismo habría un caso de confusión absoluta donde las personas que trabajan en la organización no sabrían claramente qué tareas realizar. Además, las metas y objetivos de la organización cuando no están claramente definidos por alguien en una posición de autoridad, es difícil para los empleados de la organización tener un sentido de dirección y trabajar hacia metas y objetivos comunes (Ryan, 2014).

En la siguiente Tabla 1 se especifican los principios que conforman esta gerencia.

Tabla 1.

Principios de la Gestión Administrativa

\begin{tabular}{ll}
\hline Principios & Descripción \\
\hline $\begin{array}{l}\text { Principios de } \\
\text { división del trabajo y } \\
\text { especialización: }\end{array}$ & $\begin{array}{l}\text { Todo trabajo debe ser dividido con el fin de permitir la especialización de } \\
\text { las personas en alguna actividad. Esto significa que toda persona debe } \\
\text { cumplir una sola función. La especialización provoca la división del trabajo } \\
\text { y, consecuentemente una especialización de las tareas o bien llamada la } \\
\text { heterogeneidad dentro de la empresa. }\end{array}$ \\
\hline Principios de autoridad & $\begin{array}{l}\text { Autoridad es el derecho de dar órdenes y exigir obediencia, cuestiones } \\
\text { y responsabilidad: }\end{array}$ \\
$\begin{array}{l}\text { indispensables en las actividades administrativas. La autoridad emana del } \\
\text { superior hacia el subordinado, mientras que la responsabilidad emana del } \\
\text { subordinado hacia el superior. Ambas deben ser equivalentes y equilibradas. } \\
\text { Las líneas de este principio es que debe haber una línea de autoridad y } \\
\text { responsabilidad claramente definida. }\end{array}$ \\
\hline Principios de jerarquía & $\begin{array}{l}\text { La jerarquía representa el volumen de autoridad y responsabilidad de cada } \\
\text { persona u organismo en la empresa. A medida que se asciende en la escala } \\
\text { jerarquía aumenta el volumen de autoridad y de responsabilidad. }\end{array}$ \\
\hline
\end{tabular}




\begin{tabular}{ll}
\hline $\begin{array}{l}\text { Principios de la unidad } \\
\text { de mando: }\end{array}$ & $\begin{array}{l}\text { Cada persona debe subordinarse solamente a un superior. Es el principio } \\
\text { de autoridad única, que busca evitar confusiones u órdenes dobles. Cada } \\
\text { subordinado debe tener solo un jefe. }\end{array}$ \\
\hline $\begin{array}{l}\text { Principio de amplitud } \\
\text { administrativa: }\end{array}$ & $\begin{array}{l}\text { Cada superior debe tener una cantidad adecuada de subordinados, o sea un } \\
\text { número conveniente de personas a mandar. }\end{array}$ \\
\hline
\end{tabular}

Fuente: Información adaptada de Montes (2017).

\section{Presupuesto como instrumento de control}

El proceso administrativo conlleva una serie de operaciones independientes empleadas por la parte administrativa de una institución, para el desarrollo de las acciones de planificación, organización, dirección y control. Dentro de estas actividades se encuentra el registro presupuestal, el cual puede ser descrito como el conjunto de procedimientos y recursos utilizados para planear, coordinar y controlar, a través de presupuestos, todas las operaciones y funciones de una institución, con la finalidad de conseguir el rendimiento máximo posible. De esta manera, el presupuesto representa una herramienta importante de planeación y control financiero donde se presentan de forma ordenada y en términos monetarios aquellos resultados previstos de un proyecto, plan o estrategia. Dicho en otras palabras constituye un plan financiero para estimar los ingresos y gastos de un período específico dado, está relacionado íntimamente con la planeación y se encuentra direccionado hacia el futuro y no hacia el pasado, pudiendo referirse a cualquier lapso de tiempo (años, meses, semanas y días) (Navarro, 2018).

Por otro lado, la implementación de un sistema efectivo de presupuesto representa en la actualidad una de las claves del éxito de cualquier institución, de esta manera, desde una perspectiva financiera, si no se cuenta con un procedimiento debidamente coordinado, la parte administrativa tendrá únicamente una idea empírica en relación hacia dónde se dirige la institución, dado que un sistema presupuestal constituye un instrumento de gestión para conseguir el uso más productivo de los recursos.

En el ámbito local, para lograr una óptima ejecución del presupuesto es necesario considerar varios aspectos, entre los cuales se puede destacar, por ejemplo, la gestión administrativa, el conocimiento de cada uno de los funcionarios involucrados en el proceso, el cumplimiento de los procedimientos establecidos y los objetivos del manual de puestos del Ministerio de Educación.

\subsection{Ciclo presupuestario}

Podemos indicar que el ciclo presupuestario comprende el conjunto de acciones destinadas a la óptima utilización de los recursos financieros y materiales asignados dentro de un presupuesto general con la finalidad de conseguir bienes, servicios y obras en cantidad, calidad previstos en el mismo.

Dentro del contexto nacional, según el Ministerio de Economía y Finanzas (2018) el ciclo presupuestario es definido como la programación y la clausura de la ejecución presupuestaria, el mismo que se encuentra estructurado por las siguientes fases: a) programación, donde se determinan las metas y objetivos en la planificación del Estado que se establece para un 
período de cuatro años por cuanto es denominado también como presupuesto Plurianual, b) formulación, donde se articulan las propuestas de acción y producción de los entes demandantes de recursos fiscales con los catálogos y clasificadores presupuestarios, c) aprobación, donde las instituciones del sector público no financiero cuyos presupuestos conforman el Presupuesto General del Estado, remitirán al Ministerio de Economía y Finanzas, hasta el 30 de junio de cada año, las proformas presupuestarias institucionales para su análisis y aprobación, con el fin de integrarlas y consolidarlas en la Proforma del Presupuesto General del Estado que será puesta a consideración del Presidente de la República, previo a su envío a la Asamblea, d) ejecución, que constituye el conjunto de acciones destinadas a la utilización de los recursos humanos, materiales y financieros asignados en el presupuesto con el propósito de obtener los bienes y servicios en la cantidad, calidad y oportunidad previstos en el mismo, e) seguimiento y control, que consiste en una medición de resultados físicos y financieros obtenidos y los efectos que estos han producido; así como el análisis de variaciones financieras, f) clausura, los presupuestos del sector público se cerrarán el 31 de diciembre de cada año. Después de esa fecha los ingresos que perciban se considerarán como del presupuesto vigente al momento que se cobren, después del 31 de diciembre no se podrán contraer compromisos ni obligaciones que afecten al presupuesto del ejercicio fiscal anterior, y g) liquidación, la cual refleja los resultados definitivos que dan cuenta del uso de los recursos por parte del Gobierno Central durante el año (Mendoza et al., 2018).

\section{Metodología}

El enfoque de investigación dentro del presente estudio fue de carácter cuantitativo, dado que se fundamenta en la recolección de datos numéricos derivados de cifras o cantidades implícitas en las variables de estudio, a través de la utilización de operaciones estandarizadas y admitidos por la colectividad científica (Hernández \& Mendoza, 2018).

Mediante la recolección de información se obtuvo el informe de rendición de cuentas de la Coordinación Zonal de Educación - Zona 3 durante el período enero - diciembre 2018, el mismo que fue sometido a un análisis estadístico descriptivo donde se determinó el porcentaje de ejecución del presupuesto asignado a este rubro.

Por otro lado, en relación a la modalidad de investigación fue necesario desarrollar una indagación bibliográfica, pues esta constituye una etapa indispensable en cualquier tipo de proyecto de investigación y debe garantizar la obtención de la información más relevante en el campo de estudio, de un universo de documentos que puede ser muy extenso (Gómez, Fernando, Aponte y Betencourt, 2014). En consiguiente, se procesó la información recabada en distintas fuentes secundarias; es decir, libros, revistas, estudios realizados con anterioridad, entre otros documentos privados y públicos, los mismos que fueron procesados en su totalidad escogiendo aquella con mayor relevancia y que se halla descrita a lo largo del presente manuscrito.

A su vez, en cuanto al alcance del estudio, este se centró en un nivel descriptivo, dado que consiste en el registro, análisis e interpretación de la naturaleza actual y la composición o procesos de los fenómenos. En este sentido, dentro de la presente investigación fue necesario describir los elementos de la gestión administrativa, acciones correctivas sobre el destino de los recursos 
y la capacidad de gestión, asimismo en relación a la ejecución presupuestaria se describieron los valores totales asignados, valores codificados y valores devengados de esta manera se determinó el porcentaje de ejecución en la Coordinación Zonal de Educación - Zona 3, versus el recurso recibido por la planta central o también llamado Ministerio de Educación.

Para efectos del trabajo la población se encuentra estructurada por la Coordinación Zonal de Educación, Zona 3, dado que es la institución encargada del proceso de ejecución presupuestaria en las provincias de Tungurahua, Cotopaxi, Chimborazo y Pastaza. Se puede acotar que para el desarrollo de la investigación fue necesario utilizar el informe de rendición de cuentas de la institución correspondiente al período enero - diciembre del año 2018.

\section{Resultados}

La Coordinación Zonal de Educación - Zona 3, al 31 de diciembre de 2018 en su Plan Operativo Anual registró un presupuesto codificado de USD \$38'837.144,12 y devengó un monto de USD $\$ 37 ’ 234.481,75$ logrando el $96 \%$ de ejecución presupuestaria (Figura 1). En este mismo contexto, se evidencia que a esta fecha se registra un saldo disponible de USD $\$ 34.763,15$, es decir, el $0,09 \%$ del total codificado. A continuación, en la Tabla 2 se presenta el detalle de los rubros anteriormente mencionados:

Tabla 2.

Plan Operativo Anual (POA) Coordinación Zonal de Educación Zona 3

\begin{tabular}{|c|c|c|c|c|}
\hline 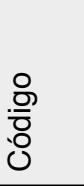 & 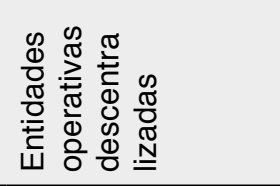 & 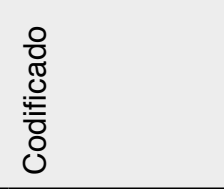 & $\begin{array}{l}\frac{0}{0} \\
\frac{\pi}{0} \\
\frac{D}{0} \\
\mathbb{1} \\
0\end{array}$ & $\begin{aligned} & \frac{\overline{0}}{0} \\
\frac{0}{0} & \frac{0}{0} \\
\circ & 0\end{aligned}$ \\
\hline 23 & Zona 3 & $\begin{array}{l}\$ \\
512.911,89\end{array}$ & $\begin{array}{l}\$ \\
508.421,17\end{array}$ & $99 \%$ \\
\hline 6647 & 16D01 Pastaza & $\begin{array}{l}\$ \\
\text { 3'557.995,35 }\end{array}$ & $\begin{array}{l}\$ \\
3^{\prime} 484.710,81\end{array}$ & $98 \%$ \\
\hline 6648 & 16D02 Arajuno & $\begin{array}{l}\$ \\
846.431,43\end{array}$ & $\begin{array}{l}\$ \\
816.654,34\end{array}$ & $96 \%$ \\
\hline 6649 & 05D01 Latacunga & $\begin{array}{l}\$ \\
\text { 3'528.419,32 }\end{array}$ & $\begin{array}{l}\$ \\
\text { 3'500.888,67 }\end{array}$ & $99 \%$ \\
\hline 6650 & 05D02 La Maná & $\begin{array}{l}\$ \\
1 ’ 770.578,77\end{array}$ & $\begin{array}{l}\$ \\
1 ' 765.770,35\end{array}$ & $100 \%$ \\
\hline 6651 & 05D03 Pangua & $\begin{array}{l}\$ \\
642.543,53 \\
\end{array}$ & $\begin{array}{l}\$ \\
641.985,00 \\
\end{array}$ & $100 \%$ \\
\hline 6652 & $\begin{array}{l}\text { 05D04 Pujilí - } \\
\text { Saquisilí }\end{array}$ & $\begin{array}{l}\$ \\
\text { 3'668.488,67 }\end{array}$ & $\begin{array}{l}\$ \\
\text { 3'029.024,77 }\end{array}$ & $83 \%$ \\
\hline 6653 & 05D05 Sigchos & $\begin{array}{l}\$ \\
778.157,77\end{array}$ & $\begin{array}{l}\$ \\
764.270,25\end{array}$ & $98 \%$ \\
\hline 6654 & 05D06 Salcedo & $\begin{array}{l}\$ \\
896.637,50\end{array}$ & $\begin{array}{l}\$ \\
893.196,65\end{array}$ & $100 \%$ \\
\hline
\end{tabular}




\begin{tabular}{|c|c|c|c|c|}
\hline 6655 & 18D01 Ambato 1 & $\begin{array}{l}\$ \\
\text { 3'854.505,85 }\end{array}$ & $\begin{array}{l}\$ \\
\text { 3'757.099,73 }\end{array}$ & $97 \%$ \\
\hline 6656 & 18D02 Ambato 2 & $\begin{array}{l}\$ \\
3,531.451,15\end{array}$ & $\begin{array}{l}\$ \\
\text { 3’422.145,66 }\end{array}$ & $97 \%$ \\
\hline 6657 & $\begin{array}{l}\text { 18D03 Baños de } \\
\text { Agua Santa }\end{array}$ & $\begin{array}{l}\$ \\
519.172,25\end{array}$ & $\begin{array}{l}\$ \\
518.024,65 \\
\end{array}$ & $100 \%$ \\
\hline 6658 & $\begin{array}{l}\text { 18D04 Patate } \\
\text {-Pelileo }\end{array}$ & $\begin{array}{l}\$ \\
1 ' 591.066,43\end{array}$ & $\begin{array}{l}\$ \\
1 ' 578.348,34\end{array}$ & $99 \%$ \\
\hline 6659 & $\begin{array}{l}\text { 18D05 Santiago de } \\
\text { Píllaro }\end{array}$ & $\begin{array}{l}\$ \\
\text { 1'019.454,31 }\end{array}$ & $\begin{array}{l}\$ \\
1 ' 018.746,34\end{array}$ & $100 \%$ \\
\hline 6660 & $\begin{array}{l}\text { 18D06 Cevallos a } \\
\text { Tisaleo }\end{array}$ & $\begin{array}{l}\$ \\
954.203,64\end{array}$ & $\begin{array}{l}\$ \\
949.109,65\end{array}$ & $99 \%$ \\
\hline 6661 & $\begin{array}{l}\text { 06D01 Chambo - } \\
\text { Riobamba }\end{array}$ & $\begin{array}{l}\$ \\
\text { 3'667.760,87 }\end{array}$ & $\begin{array}{l}\$ \\
\text { 3’098.179,00 }\end{array}$ & $84 \%$ \\
\hline 6662 & $\begin{array}{l}\text { 06D02 Alausí - } \\
\text { Chunchi }\end{array}$ & $\begin{array}{l}\text { \$ } \\
\text { 2'088.192,33 }\end{array}$ & $\begin{array}{l}\text { \$ } \\
2^{\prime} 084.362,64\end{array}$ & $100 \%$ \\
\hline 6663 & $\begin{array}{l}\text { 06D03 Cumandá - } \\
\text { Pallatanga }\end{array}$ & $\begin{array}{l}\$ \\
972.174,21\end{array}$ & $\begin{array}{l}\$ \\
967.464,64\end{array}$ & $100 \%$ \\
\hline 6664 & $\begin{array}{l}\text { 06D04 Colta - } \\
\text { Guamote }\end{array}$ & $\begin{array}{l}\$ \\
\text { 3'690.184,42 }\end{array}$ & $\begin{array}{l}\$ \\
\text { 3'690.138,63 }\end{array}$ & $100 \%$ \\
\hline 6665 & $\begin{array}{l}06 \mathrm{D} 05 \text { Guano - } \\
\text { Penipe }\end{array}$ & $\begin{array}{l}\$ \\
746.814,40\end{array}$ & $\begin{array}{l}\$ \\
745.940,46\end{array}$ & $100 \%$ \\
\hline Total & & $\begin{array}{l}\$ \\
38,837.144,09\end{array}$ & $\begin{array}{l}\$ \\
37^{\prime} 234.481,75\end{array}$ & $96 \%$ \\
\hline
\end{tabular}

Fuente: elaboración propia (2020)

También se establecieron los siguientes parámetros para la evaluación de la ejecución presupuestaria (Tabla 3) en la Coordinación Zonal de Educación - Zona 3.

Tabla 3.

Parámetros de evaluación de la ejecución presupuestaria

\begin{tabular}{ll}
\hline $\begin{array}{l}\text { Parámetros en \% de } \\
\text { ejecución }\end{array}$ & $\begin{array}{l}\text { Nivel de } \\
\text { Calificación }\end{array}$ \\
\hline $90 \%-100 \%$ & Óptimo \\
\hline $70 \%-89 \%$ & Mediano \\
\hline $50 \%-69 \%$ & Bajo \\
\hline $0 \%-49 \%$ & Crítico \\
\hline
\end{tabular}

Fuente: elaboración propia (2020) 


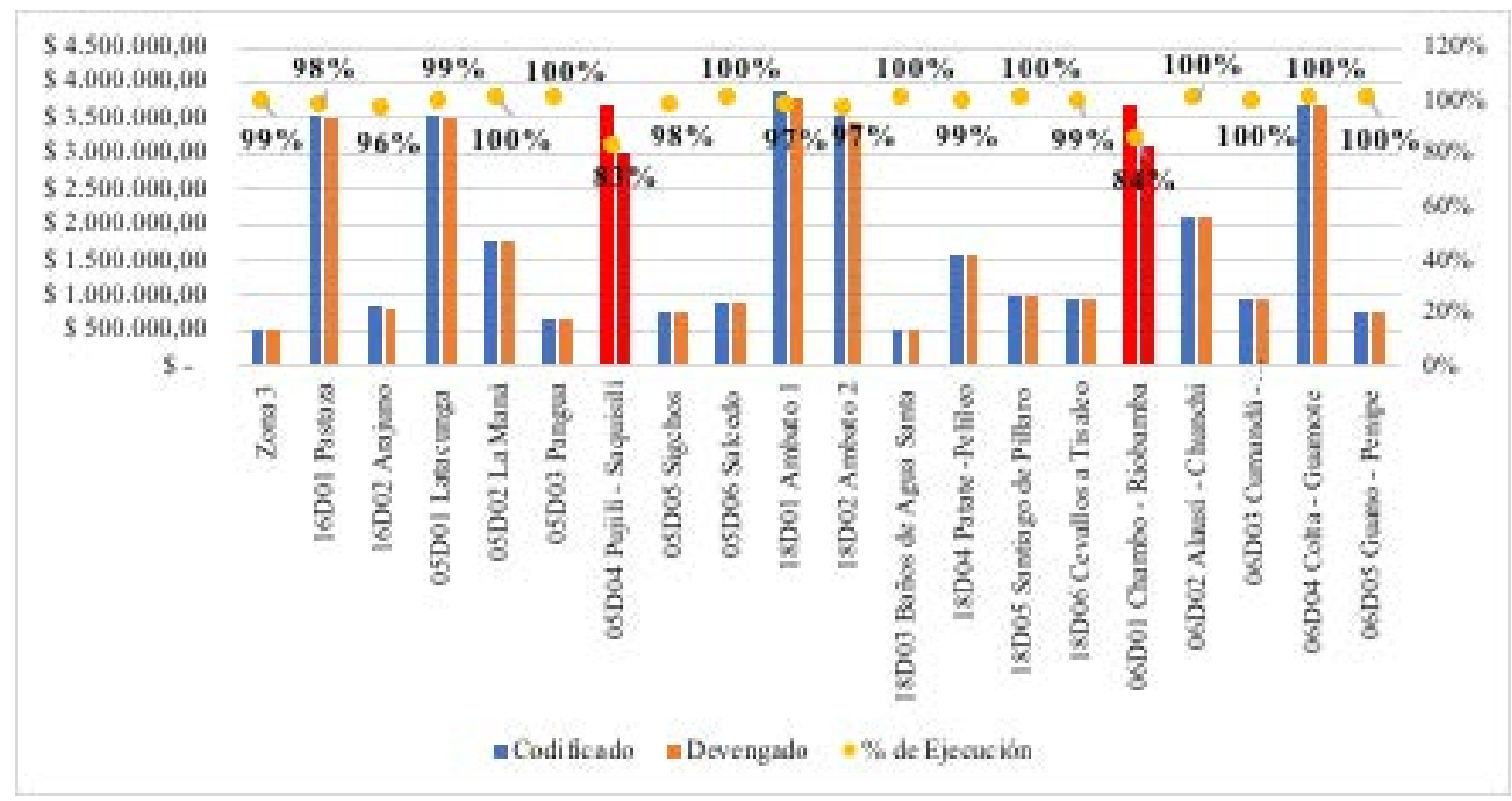

Figura 1. Ejecución presupuestaria de la Coordinación Zonal de Educación Zona 3

Fuente: Elaboración propia (2020)

A través del análisis de la ejecución presupuestaria (Figura 1), para el año 2018 se verifica que no se ha ejecutado la totalidad de los valores asignados hacia la Coordinación Zonal de Educación - Zona 3 en las diferentes Entidades Operativas Descentralizadas (EOD) dado que la suma del saldo devengado en ciertas EOD no alcanza a equiparar el saldo codificado, por ende, el porcentaje ejecutado no alcanza el $100 \%$, al tener este escenario, se deduce ciertas falencias en el ámbito administrativo que impide una correcta distribución y ejecución del presupuesto asignado. Como se mencionó en la Tabla 3, la calificación de parámetros de ejecución toma en consideración distintos escenarios; sin embargo, una vez analizados cada una de las Entidades Operativas Descentralizadas que conforman la Coordinación Zonal de Educación - Zona 3, se encuentran en un nivel óptimo de ejecución puesto que el porcentaje obtenido en cada uno de ellos se enmarca entre el $90 \%$ y $100 \%$ de ejecución, mientras que los circuitos 05D04 Pujilí - Saquisilí y 06D01 Chambo - Riobamba presentan un $83 \%$ y $84 \%$ de cumplimiento enmarcándose de acuerdo a la tabla de calificación de parámetros de ejecución en un nivel mediano $(70 \%$ - 89\%).

En este mismo ámbito de análisis, se puede manifestar que la Coordinación Zonal de Educación - Zona 3, durante el año 2018, ha ejecutado el $96 \%$ del presupuesto total asignado; dado que, se le asignó un valor total de \$38'837.144,09 de los cuales únicamente devengó un valor total de \$37'234.481,75 por lo que su gestión se ha enmarcado dentro de un nivel de asignación presupuestaria óptima.

Por parte, es importante mencionar que en un estudio similar, realizado en el año 2015 , la institución en mención determinó niveles críticos y bajos en la ejecución presupuestaria en los años 2012, 2013 y 2014, dado que se identificaron ciertos montos que fueron asignados más sin embargo nunca fueron destinados hacia las diferentes actividades, por lo que al momento de realizar el análisis correspondiente se verificó que no se ejecutaron la totalidad de los valores 
asignados para los diferentes programas ya que la suma del pagado es igual a cero, por ende el valor ejecutado es 0\% (Gavilanez, 2016).

\section{Conclusiones}

Una vez finalizada la presente investigación, se puede concluir con los datos analizados que la Dirección Técnica Zonal Administrativa Financiera de la Coordinación Zonal de Educación - Zona 3; debe disponer el seguimiento y análisis diario a la ejecución del presupuesto e informar a cada Dirección Zonal los saldos disponibles dentro de cada programa, y posterior a ello proceder a solicitar la ejecución del presupuesto que aún esté disponible con la finalidad de cumplir con la asignación del 100\% del presupuesto.

Asimismo, y de forma general, se puede indicar que la Coordinación Zonal de Educación Zona 3, durante el año 2018 ha ejecutado el 96\% del presupuesto total asignado; dado que, se le asignó un valor total de \$38'837.144,09 de los cuales únicamente devengo un valor total de $\$ 37$ '234.481,75, por lo que su gestión se ha enmarcado dentro de un nivel de asignación presupuestaria óptima.

Por otro lado, resulta necesario realizar una socialización de los recursos recibidos por parte de la Planta Central del Ministerio de Educación a cada Dirección Técnica Zonal, recordando los procedimientos desde que se genera la necesidad, solicitud de certificación presupuestaria, solicitud de compromiso, producido hasta el proceso final del pago y demás procesos administrativos.

Finalmente, es necesario tomar en consideración que un presupuesto adecuado no puede reemplazar una buena administración, como tampoco puede corregir los errores cometidos por una dirección poco organizada, no obstante, puede conseguir un trabajo gerencial más eficaz, sistemático y coordinado, dado que en la actualidad los presupuestos son empleados como un modelo matemático, el mismo que permite tomar previsiones tanto financieras, como económicas, dentro del ejercicio operativo de cualquier tipo de institución. 


\section{Referencias}

Abdulrahim, A. A., \& Elbashir, K. H. (2016). The impact of administrative management's quality on improving local government in Khartoum an applied study on Karary Locality. European Journal of Business and Management, 8(26), 94-104.

Armijo, M. (2018). Planificación estratégica e indicadores de desempeño en el Sector Público.

Bonilla Jurado, D., Delgado Salcedo, N., \& Fajardo Aguilar, G. (2020). Branding, un elemento necesario del marketing estratégico en la Cámara de Comercio de Ambato. Revista Eruditus, 1(2), 9-26. https://doi. org/10.35290/re.v1n2.2020.278

Bonilla Jurado, D., Oña Sinchiguano, B., \& López Núñez, H. (2018). Medición de innovación tecnológica como eje central del crecimiento empresarial familiar del sector carrocero de la Provincia de Tungurahua. Revista Lasallista de Investigación, 15(2), 271-285. https://doi.org/10.22507/rli.v15n2a21

Edwards, R. (2018). An elaboration of the administrative theory of the 14 principles of management by Henri Fayol. International Journal for Empirical Education and Research, 1(1), 41-52.

Escobar Mayorga, D., Mora Aristega, J., \& Huilcapi Masacón, M. (2017). Gestión administrativa y financiera en el cumplimiento presupuestario de las instituciones públicas. Polo Del Conocimiento, 2(6), 26-41. https:// doi.org/10.23857/pc.v2i6.126

Falconi Piedra, J. F., Luna Altamirano, K. A., Sarmiento Espinoza, W. H., \& Andrade Cordero, C. F. (2019). Gestión administrativa: Estudio desde la administración de los procesos en una empresa de motocicletas y ensamblajes. Visionario Digital, 3(2), 155-169. https://doi.org/10.33262/visionariodigital.v3i2.406

García, S. L. A., Vejar, M. L. L., \& Paguay, S. L. G. (2018). Alternativa Metodológica Para El Desarrollo De La Competencia Comunicativa Oral En Idioma Inglés. European Scientific Journal, ESJ, 14(5), 160. https:// doi.org/10.19044/esj.2018.v14n5p160

Gavilanez Cartagena, M. A. (2016). La gestión administrativa y su impacto en el cumplimiento de la ejecución presupuestaria de la Coordinación Zonal de Educación Zonal 3. Universidad Técnica de Ambato.

Gómez Luna, E., Fernando Navas, D., Aponte Mayor, G., \& Betancourt Buitrago, L. A. (2014). Metodología para la revisión bibliográfica y la gestión de información de temas científicos, a través de su estructuración y sistematización. Dyna, 81(184), 158-163.

Hernández-Sampieri, R., \& Torres, C. P. M. (2018). Metodología de la investigación (Vol. 4). México^ eD. F DF: McGraw-Hill Interamericana.

Marco, F., Loguzzo, H., \& Varela, F. (2016). Introducción a la gestión y administración en las organizaciones 
(Unaj (ed.)). Unaj.

Mendoza, W., Loor, V., Salazar, G., \& Nieto, D. (2018). La asignación presupuestaria y su incidencia en la administración Pública. In Ministerio de Economía y Finanzas. https://doi.org/10.23857/dom.cien.pocaip.2018. vol.4.n.3.313-33

Ministerio de Economía y Finanzas. (2018). Normas Técnicas de Presupuesto (pp. 1-53). Ministerio de Economía y Finanzas.

Montes, D. D. C. (2017). Gestión administrativa en el desarrollo comercial de la empresa creaciones Diana Carolina de la ciudad de Quevedo provincia de Los Ríos. Universidad Regional Autónoma de los Andes.

Mora, E., Bonilla, D., Núñez, L., \& Sarmiento, J. (2018). Inadaptabilidad de los docentes al manejo de plataformas virtuales: caso educarecuador. Revista Pedagógica de La Universidad de Cienfuegos, 14(62), 1-5.

Navarro, H. J. (2018). El presupuesto como herramienta de gestión y sus efectos en la estrategia de las empresas agroindustriales de la Región ICA, 2014 - 2017. Universidad de San Martín de Porres.

Pacheco Granados, R., Robles Algarín, C., \& Ospino Castro, A. (2018). Análisis de la gestión administrativa en las instutuciones educativas de los niveles de básica y media en las zonas rurales de Santa Marta, Colombia. Información Tecnológica, 29(5), 259-266. https://doi.org/10.4067/S0718-07642018000500259

Ryan Norman, S. (2014). Critical evaluation of Henry Fayol's principles of management. Advances in Economics and Business Management, 1(2), 114-118.

Solano Palapa, N., Merino Viazcán, J., \& Uc Muñoz, L. A. (2016). Aplicación del proceso administrativo en empresas de la región mixteca poblana, para contribuir al desarrollo de las áreas económico-administrativas. Revista Administración Y Finanzas, 3(7), 1-9. 
Copyright (c) 2020 Tupac Amaru Masaquiza Jerez, Adrián Marcelo Palacios Ocaña y Klever Armando Moreno Gavilánes

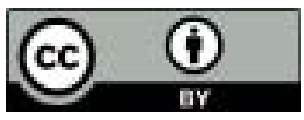

Este texto está protegido bajo una licencia internacional Creative Commons 4.0.

Usted es libre para Compartir-copiar y redistribuir el material en cualquier medio o formato

- y Adaptar el documento - remezclar, transformar y crear a partir del material-para cualquier propósito, incluso para fines comerciales, siempre que cumpla las condiciones de Atribución. Usted debe dar crédito a la obra original de manera adecuada, proporcionar un enlace a la licencia, e indicar si se han realizado cambios. Puede hacerlo en cualquier forma razonable, pero no de forma tal que sugiera que tiene el apoyo del licenciante o lo recibe por el uso que hace de la obra.

\section{$\underline{\text { Resumen de licencia - Texto completo de la licencia }}$}

